\title{
Effect of 6 Weeks of Preparation Training on Lipid Profile of High School Male Volleyball Players
}

\author{
Abbass Ghanbari-Niaki (PhD) \\ Department of Exercise Biochemistry, \\ Faculty of Physical Education and \\ Sport Sciences, University of \\ Mazandaran, Baboulsar, Mazandaran, \\ Iran \\ Fatemeh Islami (MSc) \\ Department of Faculty of Humanities, \\ School of Physical Education, \\ Golestan University, Gorgan, Iran \\ Ramezanali Arabameri (MSc) \\ Master of Physical Education, \\ Department of Education, Minoodasht, \\ Golestan, Iran \\ Aliakbar Sharifiyan (MSc) \\ Master of Physical Education, \\ Department of Education, Gorgan, \\ Golestan, Iran \\ Corresponding author: Fatemeh \\ Islami \\ Address: Deptartment of Faculty of \\ Humanities, School of physical \\ education, Golestan University, \\ Gorgan, Iran \\ Email: fatiislami@yahoo.com \\ Tel: +989111789931
}

Received : 10 Feb 2016

Revised: 01 Apr 2016

Accepted: 09 Apr 2016

\begin{abstract}
Background and Objectives: The aim of the present study was to investigate the effect of six weeks of volleyball training on lipid and lipoprotein profiles of elite high school male volleyball players in the Golestan Province (Iran) during preparation season for an international championship in France.

Methods: Training group included 12 elite volleyball players in students' premier league and control group included 12 healthy non-athlete male students. The training group practiced at $60-85 \%$ of maximum heart rate for six weeks, 90 minutes per session, six sessions per week and three sessions a day (first session: technical practice, second session: tactical practice and third session: technical/tactical practice). Blood samples were taken after 12 hours of fasting both before and after the training.

Results: Level of total cholesterol (TC), high-density lipoprotein-cholesterol (HIL-C), low-density lipoprotein-cholesterol (LDL-C) and LDL-C/HDL-C increased in the training group $(\mathrm{P}>0.05)$. However, there was a significant difference between the two groups in TC and LDL-C levels $(\mathrm{P}<0.05)$. Level of triglyceride, VLDL, and TC/HDL-C reduced in the training group after six weeks of training $(\mathrm{P}>0.05)$.

Conclusion: 0ur results show that the six-week volleyball training during the preparation season can cause abnormalities in plasma lipid profile.

Keywords: Lipid, Lipoprotein, Volleyball Player.
\end{abstract}




\section{INTRODUCTION}

There is a close association between cardiovascular disease (CVD) and lipid profile. Several clinical trials have shown the positive and negative relationships of CVD with concentration of low-density lipoprotein cholesterol (LDL-C) and high-density lipoprotein cholesterol (HDL-C), respectively (1). In volleyball, the pre-season is a period of heavy training aiming to develop technical and tactical skills and adequate physical fitness for competitions in the following season (2). In school competitions, there is limited preparation time since students' matches are held during the school season. Therefore, preparation trainings are performed with high volume and intensity in a very short time. This imposes notable physical and psychological pressure on the players and changes some biochemical parameters. This makes it difficult to establish the effects of training on lipid profile (LP) of professional volleyball players because apart from the personal characteristics of each player, particular features of trainings, especially those focused on competition, can substantially modify LP (3). Ruiz et al. stated that volleyball causes a lot of physical stress, which lower levels of undesirable plasma lipids and lipoproteins more than in less stressful sports (4). Witek et al. suggested that changes in the LP over the course of a season could be regarded as transient, with no impact on risk of CVD, because the lipid levels remained within normal physiological ranges (5). Since there are many contradictory data in this regard, we aimed to evaluate potential changes in the LP that might be induced by six weeks of training in male volleyball players of an elite high school.

\section{MATERIAL AND METHODS}

This quasi-experimental study with pretest posttest design was performed on training and control group. The training group included 12 randomly selected elite high school male volleyball players who were also the member of the Iran national team. In addition, 12 healthy high school students who were not volleyball players were randomly selected as the control group. Prior to the study, the necessary permissions were obtained from the Department of Education and informed consents were obtained from the participants and their parents. Body weight was measured in $\mathrm{Kg}$ using a SECA scale, to the nearest 0.1 $\mathrm{kg}$. Height was measured using a stadiometer to the nearest $0.5 \mathrm{~cm}$. Body mass index (BMI) was then calculated using the formula body weight $/$ height $^{2}\left(\mathrm{Kg} / \mathrm{m}^{2}\right)$. All players were on a balanced diet supervised by a dietician throughout the study. The study protocol was approved by the local ethics committee. The experimental procedures, associated risks, and benefits were explained to eligible players before participation in the study. The training group took part in preparation training for participation in an international school championship in France. The training was performed for six weeks, six days a week, three sessions a day (first session: technical practice, second session: tactical practice and third session: technical/tactical practice) for 90 minutes per session. In the first week, the participants trained at 50-60\% of maximum heart rate (MHR). They trained at $60-65 \%$ of MHR in the second week and at $70-75 \%$ of MHR in the third week. The trainings in the fourth, fifth and sixth week were performed at $80-85 \%, 70-75 \%$ and $60-65 \%$ of MHR, respectively. Training sessions were held at 89.30 and 10.30-12 A.M and 16-18 P.M. The participants warmed up for 10 minutes and then cooled down for 15 minutes after the main volleyball training. To adjust the training intensity and while-training heart rate, MHR was calculated using the following equation: MHR $=206-(0.88 \times$ age $)$. The control group was asked not to do any exercise training during the study.

Venous blood samples were taken after 12 hours of fasting from the ante-cubital fossa of the forearm, between 8.00 and 9.00 A.M. None of the players trained the day of sampling. Triglyceride (TG), total cholesterol (TC) and HDL-cholesterol (HDL-C) levels were measured by enzymatic spectrophotometric technique using an autoanalyzer (Pars Azmoon kits; Roche Diagnostics, Hitachi 912 device, Germany). These values were then used to calculate the LDL-cholesterol (LDL-C) based on the Friedewald equation: LDL-C $=(\mathrm{TC}-\mathrm{HDL}-\mathrm{C})$ - TG/5; and the atherogenic indices (TC/HDL$\mathrm{C}$ and LDL-C/HDL-C). VLDL-C was calculated using the following equation: VLDL-C $=\mathrm{TG} / 5$. Statistical analysis was performed using the IBM Statistical Package of Social Sciences (Version 20, IBM SPSS Statistics). 
Kolmogorov-Smirnov test was used to assess normal distribution of data. All data were reported as mean \pm standard deviation (SD). Paired and independent t-tests were used for within-group and between-group comparisons, respectively. Statistical significance was set at $\mathrm{P} \leq 0.05$.

\section{RESULTS}

The two groups had no significant difference in terms of age, weight, height and
BMI ( $\mathrm{P}>0.05)$. After six weeks of training, BMI significantly decreased in the training group $(\mathrm{P}<0.05)$. In addition, $\mathrm{BMI}$ was significantly different between the two groups after the training (Table 1). The level of TC, HDL-C, LDL-C/HDL-C, and LDL-C increased in the training group $(\mathrm{P}>0.05)$. The TC and LDL-C levels differed significantly between the two groups $(\mathrm{P}<0.05)$. The level of TG, VLDL, and TC/HDL-C reduced in the training group after the 6-week training $(\mathrm{P}>$ $0.05)$.

Table 1- Mean and SD of the study variables before and after six weeks of preparation training

\begin{tabular}{|c|c|c|c|c|c|}
\hline Variables & Groups & Before training & After training & $\begin{array}{c}\text { Sig. } \\
\text { (dependent t-test) }\end{array}$ & $\begin{array}{c}\text { Sig. } \\
\text { (independent t-test) }\end{array}$ \\
\hline \multirow[t]{2}{*}{ BMI $\left(\mathrm{kg} / \mathrm{m}^{2}\right)$} & Control & $19.39 \pm 3.02$ & $19.52 \pm 3.03$ & 0.075 & $* 0.000$ \\
\hline & Training & $18.14 \pm 1.35$ & $17.85 \pm 1.32$ & $* 0.000$ & \\
\hline \multirow[t]{2}{*}{ TG (mg/dL) } & Control & $136 . .83 \pm 149.24$ & $138.00 \pm 157.96$ & 0.708 & 0.625 \\
\hline & Training & $82.75 \pm 6.22$ & $76.66 \pm 50.69$ & 0.679 & \\
\hline \multirow[t]{2}{*}{ TC (mg/dL) } & Control & $148.58 \pm 15.96$ & $144.41 \pm 23.07$ & 0.172 & *0.029 \\
\hline & Training & $124.41 \pm 13.29$ & $134.25 \pm 15.70$ & 0.090 & \\
\hline \multirow[t]{2}{*}{ HDL-C (mg/dL) } & Control & $42.33 \pm 6.40$ & $42.50 \pm 8.63$ & 0.916 & 0.738 \\
\hline & Training & $42.08 \pm 8.45$ & $43.00 \pm 10.53$ & 0.574 & \\
\hline \multirow[t]{2}{*}{ LDL-C (mg/dL) } & Control & $78.83 \pm 21.88$ & $74.25 \pm 21.88$ & 0.185 & $* 0.018$ \\
\hline & Training & $64.91 \pm 14.74$ & $76.00 \pm 12.12$ & 0.056 & \\
\hline \multirow[t]{2}{*}{ VLDL-C (mg/dL) } & Control & $27.41 \pm 29.52$ & $27.66 \pm 31.55$ & 0.718 & 0.596 \\
\hline & Training & $16.58 \pm 1.16$ & $15.25 \pm 10.11$ & 0.651 & \\
\hline \multirow{2}{*}{$\begin{array}{c}\text { LDL-C/HDL-C } \\
\text { (Ratio) }\end{array}$} & Control & $1.89 \pm 0.59$ & $1.79 \pm 0.54$ & 0.465 & 0.099 \\
\hline & Training & $1.65 \pm 0.60$ & $1.85 \pm 0.44$ & 0.106 & \\
\hline \multirow[t]{2}{*}{ TC/HDL-C(Ratio) } & Control & $3.66 \pm 0.65$ & $3.50 \pm 1.24$ & 0.586 & 0.339 \\
\hline & Training & $3.16 \pm 0.83$ & $3.33 \pm 0.88$ & 0.339 & \\
\hline
\end{tabular}

* Statistical significance $(\mathrm{P} \leq \mathbf{0 . 0 5})$

\section{DISCUSSION}

After the 6-week training, BMI of participants in the training group reduced significantly. In addition, the TC and LDL levels differed significantly between the two groups. There is strong evidence that aerobic exercise is associated with favorable shifts in TG and HDL-C levels. It has been demonstrated that exercise training lower LDL-C level (6). Furthermore, independent of diet, exercise has beneficial effects on the concentration of LDLC, HDL-C, and TG (7). A recent meta-analysis showed that continuous exercise (training) induced a 5-8\% increase in HDL-C levels (8). This is attributable to increase in the activity of lecithin-cholesterol acyltransferase (LCAT), which increases the synthesis of HDL-C, and reduction in the activity of hepatic lipase, which is involved in the catabolism of these lipids. The effects of physical activity on LCAT and hepatic lipase depend on the type, intensity, frequency, and duration of the physical activity. Serum HDL-C levels are inversely associated with the risk of CVD (3). In the present study, a slight increase was observed in the levels of HDL-C in volleyball players after the 6-week training. Although this increase was not statistically significant, it should be noted that an increase of this magnitude would decrease their risk for CVD by $16-24 \%$ (8). In contrast to HDL-C, high levels of LDL-C favor the onset and development of CVD (3). This is why most studies have been focused on determination of factors that lower LDL-C levels (8). Petibois et al. (6) noted those effects in 20 rowers in a 47week study. However, HDL-C decreased in over-trained athletes. We found that the training induced an increase in LDL-C, but no similar report was found in the literature. Inconsistent with our study, in study of Mielgo-Ayuso et al.,_LP of female volleyball players improved despite having a diet high in 
fat and cholesterol. In the mentioned study, LDL-C and atherogenic indices declined, while HDL-C increased after 11 weeks of training (2). In study of Magkos et al., healthy untrained individuals were subjected to an isolated prolonged exercise (9). They found a rapid elimination of VLDL from blood and a drop in TG levels, which was associated with increased concentration of intermediate density lipoproteins (IDL), the precursor of LDL. Both IDL and LDL contain more cholesterol than VLDL. LP contains not only the serum concentrations of lipid constituents but their ratios as well. For instance, increase in TC/HDL and LDL/HDL is strongly associated with the risk of CVD and incidence of CHD in diabetics, respectively (10). Decrease in TG, VLDL and increase in TC, LDL, and LDL/HDL levels may suggest that the high-intensity training reflected by creatine kinase activity combined with some metabolic changes brought about mounting fatigue. Creatine kinase activity may serve as an indirect indicator of training loads (8) and the resulting muscle damage, which may appear several days after exercise (5). Inconsistent with our study, study of Mielgo-Ayuso et al. on female volleyball player reported a slight decrease in TC and a significant decrease in LDL-C, which indicates improvement in LP indices (3). The decrease in LDL-C in players is attributable to the effect of physical activity on skeletal muscles and activity of lipoprotein

\section{REFERENCES}

1. Boudi F Brian MD, FACP. Risk Factors for Coronary Artery Disease. Disease. 2015; http://emedicine.medscape.com/article/164163-overview. 2 . Witek K. Changes in serum lipid profile of elite volleyball players in the competition period. Biomed Hum Kinet. 2009; 1: 63-66. DOI: 10.2478/v10101-009-0016-8.

3. Mielgo- Ayuso J, Collado PS, Urdampilleta A, Miguel Martínez-Sanz J and Seco J. Changes induced by diet and nutritional intake in the lipid profile of female professional volleyball players after 11 weeks of training. Journal of the International Society of Sports Nutrition. 2013; 10(55): 1-7. DOI: 10.1186/1550-2783-10-55.

4. Tambalis K, Panagiotakos DB, Kavouras SA, Sidossis LS. Responses of blood lipids to aerobic, resistance, and combined aerobic with resistance exercise training: a systematic review of current evidence. Angiology. 2009; 60(5): 614-632.

5. Ruiz JR, Mesa JL, Mingorance I, Rodriguez-Cuartero A, Castillo MJ. Sports requiring stressful physical exertion cause abnormalities in plasma lipid profile. Rev Esp Cardiol 2004, 57(6):499-506. lipase, the enzyme responsible for hydrolyzing TG-rich lipoprotein, thereby reducing VLDL cholesterol and LDL-C. Summing up, the observed changes in LP indices are indicative of metabolic disturbances brought about by training- and competition-induced overload. Those changes may be regarded as transitory and of no significant impact on the risk of CVD.

\section{CONCLUSION}

The 6-week volleyball training in the preseason is not heart-healthy because it does not improve LP indices and increase the LDL-C, TC, and LDL-C/HDL levels. These changes may occasionally serve as an objective quantitative tool for monitoring training intensity in team sports. However, the response of these variables could be used as an objective quantitative tool for monitoring training load and better planning of training cycles throughout the season. A more comprehensive study should be performed on larger study populations to confirm our results.

\section{ACKNOWLEDGMENTS}

The authors would like to thank all participants in this study.

\section{CONFLICT OF INTEREST}

The authors declare that there is no conflict of interest.

6. Halbert JA, Silagy CA, Finucane P, Withers RT, Hamdorf PA. Exercise training and blood lipids in hyperlipidemic and normolipidemic adults: a meta-analysis of randomized, controlled trials. Eur J Clin Nutr. 1999; 53(7): 514-522.

7. Huffman KM, Hawk VH, Henes ST, Ocampo CI, Orenduff MC, Slentz CA, et al. Exercise effects on lipids in persons with varying dietary patterns-does diet matter if they exercise? Responses in Studies of a Targeted Risk Reduction Intervention through Defined Exercise I. Am Heart J. 2012; 164(1):117-124.

8. Pattyn N, Cornelissen VA, Eshghi SR, Vanhees L. The effect of exercise on the cardiovascular risk factors constituting the metabolic syndrome: a meta-analysis of controlled trials. Sports Med 2013, 43(2):121-133.

9. Mariscal-Arcas M, Romaguera D, Rivas A, Feriche B, Pons A, Tur JA, et al. Diet quality of young people in southern Spain evaluated by a Mediterranean adaptation of the Diet Quality Index-International (DQI-I). Br J Nutr 2007, 98(6):1267-1273.

10. Green PP, Namboodiri KK, Hannan P, Martin J, Owen AR, Chase GA, et al. The Collaborative Lipid Research Clinics Program Family Study. III. Transformations and covariate adjustments of lipid and lipoprotein levels. Am J Epidemiol. 1984; 119(6): 959-974. 